\title{
Le français en Algérie, création et variation comme vecteur d'adaptation : le cas de l'emprunt au berbère
}

\author{
MERZOUK, Sabrina \\ Maître de conférences au département de Français \\ Université Abderrahmane Mira, Bejaia, Algérie \\ Merzouk.sabrina@yahoo.fr
}

Pour des raisons historiques, géographiques, économiques et autres, l'Algérie est un pays marqué par une diversité linguistique qui se traduit par une co-présence sur le territoire des langues arabe, française et berbère. Cette situation de plurilinguisme est visible sur le plan des pratiques langagières du locuteur algérien. Dans son contact avec les autres langues présentes, le français se développe de façon différente en comparaison avec celui de l'Hexagone. La néologie y est pour beaucoup dans cette variation et la presse écrite est le témoin le plus sûr de la vitalité et du dynamisme que connaît le français en Algérie. La pratique de différents systèmes linguistiques permet aux différentes langues de s'enrichir les unes à partir des autres. Cet enrichissement a pour résultat une création assez dense de mots et de sens nouveaux.

\section{Problématique}

À notre époque où le terme de «mondialisation" est devenu de plus en plus à la mode, on assiste à l'abolition des frontières entre les peuples et par conséquent entre les langues aussi. Le présent article vient répondre à la question du rôle de l'emprunt dans le renouvellement lexical du français en Algérie. L'interrogation majeure à laquelle notre travail tente de répondre est la suivante : quel est l'apport de l'emprunt au berbère dans la création néologique du français dans la presse écrite en Algérie ? Les objectifs visés à travers cette étude sont les suivants : a) voir comment le locuteur arrive à répondre à ses lacunes en matière de lexique et les stratégies qu'il met en œuvre pour cela; b) déterminer l'apport de l'emprunt au berbère dans l'enrichissement du lexique français en Algérie.

\section{Les langues et leur statut en Algérie}

Dans la situation linguistique qui caractérise l'Algérie, on compte la présence de l'arabe littéral ${ }^{1}$, langue de l'école, de la religion et de l'administration judiciaire. Bien qu'il soit la langue officielle, son officialisation reste symbolique puisqu'il a l'une des places les plus réduites dans les usages institutionnels : banque, poste et télécommunications. «Si l'arabe est déclaré langue officielle pour des raisons idéologiques, son emploi dans le secteur économique et les administrations est rendu problématique par la présence des autres langues et plus particulièrement de l'arabe dialectal [...] » (Derradji, 1995 :111). Dans le cadre informel et milieu familial, l'arabe littéral cède la place au français, à l'arabe dialectal et au berbère. Ces deux derniers sont tous deux, pour les Algériens, des langues maternelles.

Le français fut introduit en Algérie par les voies de la colonisation². Les Algériens, ayant montré depuis toujours leur aptitude à l'apprentissage des langues étrangères, l'ont acquis. Celui-ci devient langue d'usage pour un bon nombre de locuteurs, ce qui classe l'Algérie dans les premiers rangs des pays francophones. Bien qu'il ait officiellement le statut de langue étrangère («[...] le français investit, en Algérie, toutes les sphères du savoir auxquelles l'arabe n'a pu encore y accéder » Zeghidour, 1994 : 375), le français est vu, par les Algériens, comme la langue de l'acquisition du savoir, de l'ouverture vers le monde extérieur et s'affirme être un outil indispensable pour le développement technologique. 
Compte tenu de sa flexibilité, l'arabe dialectal, à usage exclusivement oral, est essentiellement utilisé en milieu familial, à la télévision et à la radio. L'arabe algérien est « la langue, de loin la plus parlée par les Algériens $[\ldots]$ », elle «s'est imposée comme langue véhiculaire dans les communications interalgériennes entre locuteurs berbérophones de différentes variétés et entre ceux-ci et locuteurs arabophones " (Dourari, 2003 : 7). Cette langue se développe grâce au nombre important de locuteurs qui la parle et s'enrichit grâce aux nombreux emprunts faits aux autres langues, le berbère et le français. L'arabe dialectal se divise en parlers locaux différents de l'est à l'ouest et du nord au sud. Leur différence réside dans les particularités les caractérisant sur différents niveaux : lexical, phonologique, syntaxique et sémantique. L'arabe dialectal devient aujourd'hui un centre d'intérêt pour un bon nombre de chercheurs qui s'interrogent sur son vrai statut et sa fonction. Plusieurs articles et forums de discussions tentent de trouver une réponse à cette question. Ouardia $\mathrm{Aci}^{3}$, chercheur spécialiste en socio-didactique, résume ainsi, au terme d'une étude menée sur la vision qu'ont les Algériens des langues présentes en Algérie : « [1]'arabe classique, c'est la langue de l'école et de la religion. Le français, c'est la langue du savoir, de l'université et du colonialisme. L'arabe algérien, c'est notre langue, dialna ». De son coté Kamel Daoud ${ }^{4}$ avance à son tour: "l'algérien est une langue à part entière comme toutes les autres langues utilisées pour communiquer. On parle, on commerce, on débat, on vit, on aime en algérien ${ }^{5} »$. Dépourvu de graphie et de normes, l'arabe algérien tente peu à peu de se forger une écriture et des normes, chose constatée selon Aci : "le point de passage entre le dialecte et la langue se situe au moment où l'on donne une identité graphique à un système linguistique oral. Or cette consécration de l'algérien à l'écrit s'achemine peu à peu. Aujourd'hui, sur internet ou par texto, les gens écrivent quotidiennement en algérien. Même les campagnes publicitaires commencent à se faire dans cette langue ».

Ayant longtemps souffert de la pression de la politique d'arabisation, le berbère constitue, au même titre que l'arabe dialectal, la langue maternelle d'un bon nombre de locuteurs. C'est sans doute ce qui a permis sa préservation. Cette langue jouissait pendant longtemps d'un usage oral exclusif. Transmise par voie d'héritage oral : contes, dictons, proverbes, elle ne s'est pas laissée mourir et s'enrichit continuellement par voie d'emprunts à l'arabe et au français. On appelle souvent cette langue « le tamazight», terme qui marque l'unicité dans laquelle on dénombre plusieurs variétés telles que: kabyle, chaoui, mozabite, targui... Il faut signaler qu'à l'intérieur de ces même variétés, d'autres sous-variétés existent.

\section{La politique d'arabisation}

Après l'indépendance de l'Algérie, une certaine attitude qualifiée de "francophobie» (Maougal, 1996 :106) s'est installée chez les Algériens. Faire revivre les traditions arabo-musulmanes devient l'un des objectifs principaux de la politique algérienne de l'époque. L'objectif était donc la mise en place «d'une stratégie immédiate pour une planification linguistique particulière à l'Algérie reflétant ses origines et traditions arabo-musulmanes » (Mitchell $1996: 99$ ).

L'indépendance de l'Algérie fut donc suivie de la volonté des pouvoirs de l'époque d'instaurer une politique linguistique monolingue, en renforçant l'usage de l'arabe littéral. Usage appuyé par des textes officiels, notamment l'article 5 de la Constitution de 1963 stipulant que « la langue arabe est la langue nationale et officielle de l'État ». Les langues qui se partagent le paysage sociolinguistique algérien ont subi une attitude de rejet et d'exclusion. Car ces langues (berbère, arabe dialectal et français) sont perçues selon Laroussi (1997: 30) comme «symbole de division et constituent de ce fait une menace pour la cohésion nationale ».

\subsection{Echec de la politique d'arabisation}

Le pouvoir algérien avait donc affiché son ambition visant à répandre l'arabe standard (littéral) en le nommant langue nationale et officielle de l'État algérien. Cette politique visant à promouvoir de force 
l'arabe a établi des barrières à son expansion. Son échec est dû à sa détermination à ignorer les langues maternelles utilisées dans le quotidien des citoyens. Grandguillaume (1998: 17) notait, quant au non aboutissement de cette politique : «en réalité ces effets n'ont pas été obtenus pour diverses raisons. D’une part, son imposition forcée. D'autre part, cette politique relève plus d'une décision politique qui émane de volontaristes de la conquête du pouvoir ».

\subsection{La réaction des locuteurs berbères et l'affirmation d'une langue}

Face à cette imposition forcée de l'arabe au détriment des autres langues présentes sur le territoire algérien, les locuteurs berbères ont recouru à la revalorisation de leur langue et à l'affirmation de leur identité. «Après de longues années de silence et d'interdit, la question de la langue et de la culture berbère a enfin gagné droit d'être matière à débat» (Louanouci 1998: 137). Ce droit légitime a été arraché de force. Il est le fruit de plusieurs actions de protestation et de révolte contre certaines décisions de l'État algérien comme la suppression de la chaire berbère de l'université d'Alger en 1970, l'interdiction en 1975 du groupe de l'Académie berbère fondée en 1967. Suite à ces décisions, une série d'événements s'est succédé en Kabylie et à l'échelle nationale. Citons ceux de 1980, déclenchés suite à la répression des étudiants de l'université de Tizi Ouzou alors que ceux-ci protestaient contre l'interdiction de la conférence de Mouloud Mammeri ${ }^{6}$ par les Autorités.

Malgré la première reconnaissance semi-officielle en 1990 de la langue berbère, il y eut en 1994 la grève des cartables revendiquée par le MCB (Mouvement Culturel Berbère), réclamant le droit d'instaurer le berbère comme langue d'enseignement. En avril 2001, dans le cadre d'une commémoration des événements du Printemps berbère, des événements violents éclatèrent entraînant la mort de plusieurs citoyens, le prix à payer pour une langue berbère qui acquiert alors le statut de langue nationale ${ }^{7}$, un statut qui est loin d'assouvir l'envie de la masse amazighophone qui vise une reconnaissance du tamazight comme langue officielle au même titre que l'arabe littéral. « La concession du statut de langue nationale à l'amazigh est loin de satisfaire les amazighophiles qui exigent la co-officialité de leur langue au même titre que l'arabe et des moyens à la fois juridiques et financiers pour l'aménager et élargir aussi bien ses fonctions sociales que sa pratique au double niveau horizontal et vertical » (Chérif 2004 : 72 ).

Aujourd'hui, le berbère occupe une place importante vouée à l'optimisme. Un bon nombre d'étudiants s'inscrivent dans les universités pour étudier leur langue, notamment après la création du département de la langue et de la culture berbère. La revalorisation de la langue se fait aussi par le biais de la revalorisation de la culture elle-même. Pour faire revivre les traditions, des galas, des expositions artistiques de bijoux, de vêtements, de tapis, ainsi que des ateliers de cuisine du terroir, sont régulièrement organisés. Le berbère devient également un moyen de communication en intégrant l'audio-visuel. En effet, la chaîne II est une chaîne de radio qui a réussi, malgré les censures étatiques ayant menacé son existence, à conquérir un nombre important d'auditeurs. La création aussi des chaînes télévisées satellitaires $T V 4$ et BRTV (Berbère télévision) en langue berbère constitue une victoire.

\section{Plurilinguisme, contact de langues et alternance codique}

Pays plurilingue, l'Algérie compte la présence de quatre langues. Cette situation de plurilinguisme non institutionnalisé, visible lors des interactions verbales chez les individus, engendre, dans bien des cas, dans des situations de contacts entre les différents codes linguistiques, la création sous ses différentes formes. Car, en Algérie, une grande partie de la population maîtrise au moins deux codes linguistiques. Ce côtoiement et cet emploi de deux ou plusieurs langues de façon alternée entraîne une création lexicale assez dense et visible notamment dans le cadre que nous envisageons d'analyser : celui de la presse écrite.

En parlant français, le locuteur met en mots toute une charge culturelle de croyances, de convictions et d'idéologies ; en somme, tout un vécu qu'il tente d'exprimer dans une langue qui n'est pas la sienne. Pour 
désigner en français des référents ou des choses qui relèvent de sa réalité quotidienne, le locuteur algérien se trouve confronté à un manque de termes. Il recourt ainsi à l'emprunt, en transférant de sa langue (le berbère) des termes qu'il introduit dans des contextes exclusivement français. "La société algérienne étant plurilingue, ce contact des langues se traduit par des comportements langagiers très particuliers mais tout à fait naturels pour ce type de société. Les idiomes s'interpénètrent au gré des relations sociales, des stratégies discursives des locuteurs et de leurs compétences linguistiques et surtout en fonction du caractère formel et/ou informel de la situation de communication » (Queffélec 2002 :112). Notre étude du français d'Algérie se fait en vue d'un objectif principal qui est de montrer que le français en Algérie évolue d'une manière particulière, affecté par le lexique d'autres langues. Cet usage alterné du français et du berbère donne naissance à une forme de mélange.

\subsection{Définition de l'alternance codique}

Dans la situation linguistique que vit l'Algérie marquée par une présence linguistique diverse et variée, il est courant de repérer, sur le plan des pratiques langagières, l'usage alterné des langues en présence. Ce phénomène de mélange de codes, touchant l'arabe et le français, touche également le berbère et le français. Les définitions qui lui sont accordées sont multiples, parmi lesquelles on citera celle de Poplack (1988: 36) le considérant comme «le recours à des fragments de phrases provenant d'une langue pourvue des caractéristiques morphologiques, syntaxiques et lexicales propres à cette langue et qui viennent se juxtaposer à un fragment d'une autre langue ». Ainsi, dans un même énoncé, des passages dans deux langues peuvent s'interpénétrer. Gumperz (1982: 58), pour sa part, considère l'alternance codique «comme le juxtaposition, à l'intérieur d'un même échange verbal, de passages ou de discours appartenant à deux systèmes ou sous-systèmes grammaticaux différents ». Le même phénomène est selon Myers-Scotton $^{8}$ (2002) défini comme l'insertion, au sein de la même phrase, d'éléments d'une langue A (le berbère pour ce qui est de la présente étude) dans un entourage syntaxique déterminé et régi par les règles grammaticales de la langue $B$ (le français langue d'accueil). J. Hamers et M. Blanc considèrent que le mélange de codes « est caractérisé par le transfert d'éléments d'une langue $l y$ dans la langue de base $l x$, dans l'énoncé mixte qui en résulte on peut distinguer des segments unilingues de $l x$ alternant avec les éléments de $l y$ qui font appel à des règles des deux codes »" .

Les éléments berbères de notre corpus qui figurent dans des contextes français sont des mots simples et ne dépassent pas la dimension de la lexie simple. Tels qu'ils se présentent dans leurs contextes d'emploi, ces mots gardent tantôt les marques grammaticales de la langue d'origine (le berbère) et subissent tantôt celles de la langue d'accueil (le français) et dans un troisième cas de figure, ils subissent à la fois les règles de la langue source et celles de la langue d'accueil.

Dans ses différentes typologies, l'alternance codique peut être visible (selon Bourhis, Lepicq, Sachdev, 2000) à trois niveaux : une alternance dans laquelle deux langues sont employées dans la même phrase ; une alternance codique interphrastique, c'est-à-dire que dans un même discours, deux phrases s'alternent, l'une en berbère, l'autre en français; et, enfin, une alternance codique d'un tour de parole. Il s'agit, dans une interaction, d'échange de phrases entre locuteurs, l'un communiquant dans la langue A et l'autre dans la langue $\mathrm{B}$.

\subsubsection{Distinction entre emprunt et alternance codique}

Dans une distinction emprunt/alternance codique, Shana Poplack estime que l'établissement d'une frontière entre les deux phénomènes est difficile, notamment lorsque, dans un discours de la langue $\mathrm{B}$ apparaît une unité lexicale isolée, ayant subi à la fois les marques des langues A et B. «Mais l'étude empirique des données sur le bilinguisme nous démontre qu'il est souvent impossible de distinguer l'alternance véritable. C'est tout particulièrement le cas quand une unité lexicale isolée provenant d'une langue apparaît dans le discours de l'autre, obéissant à la fois aux règles grammaticales des deux langues » (Poplack, 1988 : 28). Il est donc important de voir quels sont les critères pouvant servir de repères de distinction entre l'emprunt et l'alternance codique. Pour Dubois (1973: 188), il y a emprunt 
linguistique quand un parler A utilise et finit par intégrer une unité ou un trait linguistique qui existait précédemment dans un parler $B$ et que $A$ ne possède pas; l'unité ou le trait emprunté sont eux-mêmes appelés emprunts ». Poplack (idem : 30-31) considère que contrairement à l'alternance « la plupart des items empruntés ont tendance à s'intégrer, du moins morphologiquement et syntaxiquement, aux structures de la langue réceptrice. Ils portent les mêmes flexions morphologiques et occupent les mêmes positions syntaxiques que leurs équivalents dans la langue réceptrice ». Alors que l'alternance, nous l'avons bien signalé à partir de la définition de Gumperz (1982), concerne deux codes qui alternent dans un même énoncé, mais chacun devant répondre à ses règles grammaticales propres. Il n'y a donc aucun mélange entre les deux systèmes. Dans une étude lexicale comme la nôtre, il est important que l'analyse soit orientée vers l'examen des marques morphologiques et syntaxiques qui peuvent révéler si le mot utilisé relève du procédé d'emprunt ou résulte d'une alternance des codes.

\section{Analyse du corpus}

Notre corpus comprend 250 lexies berbères. Celles-ci sont recueillies dans la presse écrite. Les journaux auxquels nous nous sommes référée sont : El Watan, Liberté, Le Soir D'Algérie, La Dépêche de Kabylie, La Tribune et l'Authentique. Ces mots montrent bien que le français emprunte au berbère. Ces emprunts sont des mots qui renvoient à l'univers référentiel du sujet parlant berbère algérien. Ils correspondent à des réalités spécifiques au locuteur, en rapport notamment avec ses croyances, ses coutumes et traditions.

\section{L'insertion de l'emprunt au berbère dans le contexte français}

Tout comme le calque et le xénisme, l'emprunt est un procédé qui contribue au renouvellement du lexique grâce à un échange sous forme de transfert d'unités lexicales de l'un à l'autre système linguistique. Mais contrairement au calque, qui est nécessairement traduit avant d'être intégré, et au xénisme caractérisé par la présence de marques qui soulignent que le terme n'appartient pas encore à la langue emprunteuse, l'emprunt se voit attribuer les marques d'intégration en rapport notamment avec sa prononciation (adaptation phonologique) et sa morphologie (marques de genre, nombre et formation de dérivés).

\section{Les critères d'intégration des emprunts berbères}

Les critères d'intégration des mots berbères peuvent se résumer en l'adaptation phonologique, mais également morphologique justifiée par l'attribution de l'article comme manifestation du genre accordé à l'unité lexicale ainsi que les marques de pluriel et les conjugaisons verbales. Les reprises anaphoriques qui représentent, selon Maingueneau (1996:35), «[..] la reprise d'un segment par un autre placé après », interviennent également dans l'identification du genre et du nombre attribués au mot emprunté. L'unité lexicale subit des modifications qui montrent l'appropriation de celle-ci par le système linguistique français : «tout élément phonique ou morphologique nouveau (issu de A) se modifie selon les besoins et les habitudes de la langue $\mathrm{B}$ emprunteuse qui prend comme repère les critères de pertinence et d'économie linguistique qui lui sont propres »(Cheriguen, 2002 : 74). Ces critères peuvent se résumer comme suit :

\subsection{Les marques d'intégration morphologique}

\subsubsection{Le genre}

Les noms sont généralement regroupés en deux catégories distinctes à partir du critère de genre. Ce critère de différenciation peut être dépendant du critère de sexualisation, notamment pour ce qui est de la distinction entre les êtres mâle/femelle comme dans : 
Argaz « homme » [+mâle $]$ / tamettut « femme » [+ femelle $]$.

Awtul « lièvre » [+mâle $] /$ tawtult « hase » $[+$ femelle $]$.

Mais ce critère, fonctionnant pour les êtres animés, n'est pas valable pour les non sexués (choses). En outre, le passage d'un genre à un autre en berbère ajoute au mot une charge sémantique additionnelle en rapport, notamment avec :

\section{- La dimension}

Le nom au masculin désigne généralement un référent d'une dimension plus grande par rapport à celui désigné par le même nom au féminin, formé à partir de la même racine à l'exemple de :

$$
\begin{aligned}
& \text { Afus « main »------- tafust « petite main »; } \\
& \text { Ameqyas « anneau »--------- tameqyast « petit anneau ». }
\end{aligned}
$$

Mais, il faut noter aussi que ce critère de dimension, qui entre dans la distinction, en genre, entre les noms masculins et féminins, est souvent accompagné du critère d'appréciation. Dans certains cas, l'usage du masculin dénote une péjoration par rapport à la forme féminine du même mot, ce qui est visible à partir de :

$$
\text { Tametțut « femme » ----------------- amețtu « femme virile, hommasse ». }
$$

Dans d'autres cas, c'est le passage du masculin au féminin qui entraîne la péjoration, chose perceptible à partir de :

$$
\text { Argaz « homme »----------------targazt « homme efféminé, peureux ». }
$$

\section{- Ensemble et unité}

Soit l'exemple suivant :

$$
\text { Awetțuf nom masculin « fourmi »----------------tawetțuft nom féminin « fourmi ». }
$$

En plus de la distinction en genre, le nom en question est l'expression du collectif. Ainsi tawettuft désigne une seule fourmi, alors que le mot lui-même au masculin désigne non seulement le mâle de la fourmi mais également un ensemble de fourmi, espèce.

\section{- Ressemblance}

Le signifiant féminin formé sur la base d'un nom masculin peut résulter de l'identification d'un rapprochement de forme entre les êtres (morphologie approximativement identique) sans que l'un ne soit réellement le féminin de l'autre.

$$
\text { Izi nom masculin « mouche »------------------- tizit nom féminin « moustique » }
$$

Dans cet exemple, le mot féminin tizit «moustique » est créé sur la base du nom masculin izi alors qu'il n'est pas son masculin.

\section{A. Les noms masculins berbères}

Le morphème qui indique le genre masculin en berbère est situé à l'initiale du mot, chose constatée à partir de mots comme :

$$
\underline{\boldsymbol{a}} \text { mghar [amyar] « vieux », } \underline{\boldsymbol{a r g a z}} \text { [argaz] « homme », } \underline{\boldsymbol{a}} \mathbf{g} \text { ellid [ageliḍ] « roi ». }
$$


Si cette remarque ne s'applique pas à des mots comme tvak [țvaq] «corbeille», m'qam [lemqam] «mausolée », et taleb [țaleb] ${ }^{10}$ «marabout, saint», ce n'est que parce que ceux-ci constituent des emprunts à la langue arabe.

En contexte, dans un discours en français, le genre de ces mots est identifié à partir de la présence des déterminants que sont: les articles défini et indéfini masculins, les pronoms et adjectifs possessifs et démonstratifs ainsi que l'accord avec l'adjectif qualificatif :

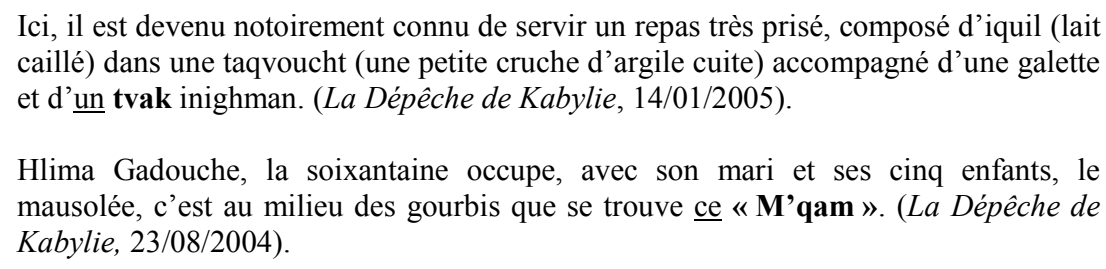

Les termes berbères empruntés apparaissent dans des contextes français accompagnés des déterminants qui indiquent leur genre. Les articles véhiculent, pour la plupart, le même genre que celui auquel répondent ces lexies en langue source (le berbère).

Ainsi, le mot amghar [amyar] utilisé dans le sens de « responsable du comité de village », singulier du pluriel ímyaren, se voit attribuer le genre masculin véhiculé par l'article le dans :

"C'est vraiment inouï" estime l'un des hommes qui fut un jour le «Amghar », responsable du comité de village. (La Dépêche de Kabylie, 14/05/2005).

\section{a. La formation plurielle des noms berbères masculins}

Formés de différentes façons, le pluriel des masculins berbères résulte non pas d'un changement radical du signifiant du mot, mais plutôt des processus suivants :

\section{- Changement du morphème initial et ajout de -en}

C'est à partir de la modification du morphème initial du mot masculin (qui est le $\boldsymbol{a}$-) et par ajout de -en que sont formés :

Iderwichen [iderwicen] de la racine berbère DRWC du singulier aderwich (masculin de taderwicht) qui signifie dans son contexte d'apparition « fou en relation avec les esprits » dans :

Ce sont, dit-on, les vrais fous iderwichen ou imeslab, qui sont en communication avec le monde de l'invisible. (La Dépêche de Kabylie, 29/08/2004).

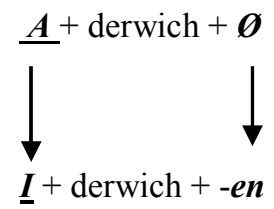

Imessebriden vient de la racine BRD pluriel de amessebrid prenant le sens de «passants ou voyageurs » dans : 
Ce fut encore un temps où des âmes charitables creusaient des puits au bord de la route pour le voyageur étanche qui a soif. Mieux, on plantait aussi des figuiers. La légendaire Tanoqalt Bavrid dont les rameaux sont à la portée de tous les Imesbriden, de tous les Imsawqen. (La Dépêche de Kabylie, 01/08/2004).

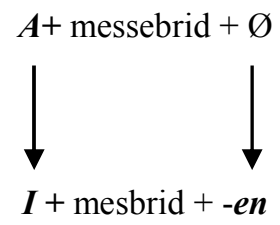

C'est à partir de ce même modèle « $\boldsymbol{i}$-..........en» que sont formés aussi : ímsawqen « approvisionneurs », imazighen "hommes libres» (nom donné aux habitants d'Afrique du nord), Ig'ezaren «boucher », ímeghriwen " fêtards », íchewiqen, « chants berbères », ifenanen " artistes », idhebbalen $«$ musiciens ».

Comme le montrent ces exemples, ces mots apparaissent dans leur contexte d'utilisation accompagnés des marques du pluriel berbère. Ils n'ont pas reçu le morphème de pluriel du français (-s).

- Pluriel formé par ajout du -an

Dans cette formation, le pluriel résulte de l'ajout d'un « Ø .........-an » à la fin du mot. C'est ainsi que sont construits Inighman du singulier inighem [iniyem] « figue sèche » dans :

Et pour ne pas déroger à la tradition, l'huile d'olive est à l'honneur ainsi que les dattes et, fruits incontournables dans la région, les fameux «inighman», «thazarth» ou figues sèches. Trempées dans l'huile d'olive, ils ont la saveur du bonheur. (Liberté, $13 / 02 / 2003)$.

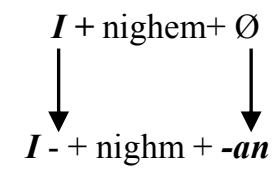

De la même façon est formé le mot $\underline{\mathbf{I}}$ thran du singulier ithri [itri, atri] « étoile, en parlant des stars du chant », igheblan du singulier aghbel [ayvel] « soucis, tracas ».

\section{- Changement du morphème initial et morphème final}

C'est la formation d'un pluriel par modification du morphème initial $\boldsymbol{a}$ - et du morphème final $\boldsymbol{-} \boldsymbol{u}$ dans le mot asefru « poème » qui donne au pluriel $\underline{i} \operatorname{sefr} \underline{a}$ dans :

$$
\downarrow_{\boldsymbol{I}-+\operatorname{sefr}+\boldsymbol{- a}}^{A+\operatorname{sefr}+\boldsymbol{u}}
$$

Sans nul doute, c'est à Mouloud Mammeri que revient le mérite d'avoir eu cette évocation sur les Isefra de Si Mohand qui, à bien des égards, ont situé le poète dans le contexte d'une société qui a connu des générations qui ont été marquées par une colonisation inhumaine. (L'Authentique, 27/12/2005). 


\title{
- Pluriel formé par modification du premier et de l'avant-dernier morphème
}

C'est à partir du l'altération du premier et de l'avant-dernier morphème qu'est formé le mot Imeslab, de la racine berbère SLB, pluriel du singulier ameslüb « fou, insensé » dans :

Ce sont, dit-on, les vrais fous, iderwichen ou imeslabb, qui sont en communication avec le monde de l'invisible. Il y a les autres fous, ceux qui parlent sans fin, apostrophant les gens, proposant de leur dire l'avenir ». (La Dépêche de Kabylie, 29/08/2004).

De amessedrar, de la racine berbère SDR, est formé le pluriel ímesdourar [imesdurar] qui signifie «montagnards ». Cette formation résulte du remplacement du $\boldsymbol{a}$ - initial par un $\boldsymbol{- i}$ et de l'insertion du -ou(marque du pluriel) dans :

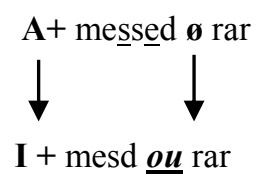

\begin{abstract}
À l'intérieur de l'école, on distingue beaucoup de visiteurs étrangers à la région mais aussi de vrais «imesdourar». Parmi les visiteurs, notre correspondant à Aïn El Hammam, qui a raflé toutes les infos sur la fête du tapis et qu'il a livré à nos lecteurs quotidiennement». (La Dépêche de Kabylie, 29/08/2004).
\end{abstract}

- Pluriel formé à partir d'un singulier berbère $+-s$ marque du pluriel français

Le signifiant du mot masculin pluriel peut résulter de l'ajout, à un singulier, de la marque du pluriel français. Ainsi, résulte de l'ajout du $-\boldsymbol{s}$ :

Isem $\underline{s}$ du singulier isem «nom » (dont le pluriel en berbère est ismawen (emprunté à l'arabe إسم marque du pluriel français dans :

\begin{abstract}
Sans cela, nous serions surpris par des situations fầcheuses, au moment ou l'on tente de restituer ce qui reste de la fraternité. Si on s'amuse à s'attaquer à tous les " ismes " la liste risquerait d'être longue et épineuse. (La Dépêche de Kabylie, 08/08/ 2004).
\end{abstract}

\section{- Le double marquage du pluriel berbère}

Certains des pluriels contenus dans notre corpus sont doublement marqués, c'est-à-dire qu'ils subissent à la fois les marques de pluriel de la langue berbère et celles du français. Ainsi, l'ajout de la marque $« \boldsymbol{i}$ ..........-an » au mot aghvel [ayvel] forme en berbère le pluriel ighevlan(e) auquel s'ajoute le -s morphème marquant le pluriel en français dans ighevlanes :

\footnotetext{
Et comme tout nouveau chanteur, dont l'intention est d'aller vers le public et "crier» par versification «Ighevlanes » de sa génération, il est d'abord passé par l'émission «Les chanteurs de demain » de Medjahed Hamid et l'appréciation de ce dernier n'a fait que l'encourager à produire. (La Dépêche de Kabylie, 08/08/2004).
}

De la même façon est formé íderwichens, du singulier aderwich [aderwic], lequel par voie d'ajout des

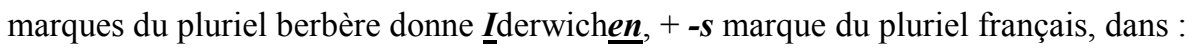

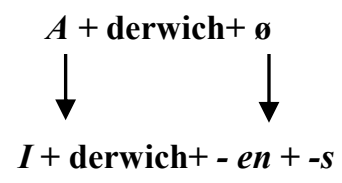


S'il fallait une dernière absurdité, ce sera celle-ci, de sources concordantes, Hassan Hattab se serait réfugié en Kabylie, pays des Iderwichens. (El Watan, 14/03/2004).

Le tableau ci-dessous résume les différentes marques que prennent les noms masculins d'origine berbère au pluriel dans les textes français : 


\begin{tabular}{|c|c|c|c|c|}
\hline \multicolumn{3}{|l|}{ 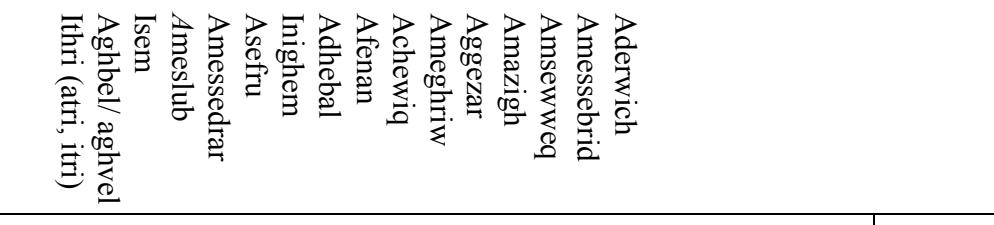 } & \multicolumn{2}{|c|}{ 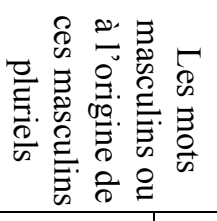 } \\
\hline \multicolumn{2}{|l|}{ 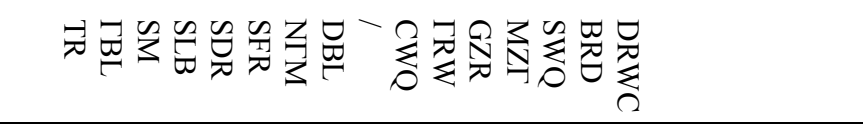 } & 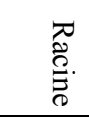 & \multirow{3}{*}{ 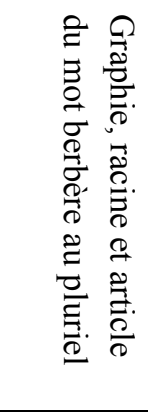 } & \multirow{10}{*}{ 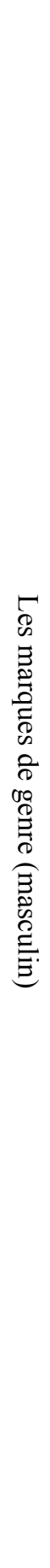 } \\
\hline 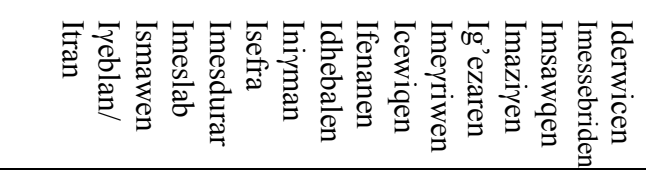 & & $\begin{array}{l}8 \\
\stackrel{8}{0} \\
0 \\
0 \\
\frac{0}{0} \\
\frac{0}{0} \\
\end{array}$ & & \\
\hline \multicolumn{2}{|l|}{ 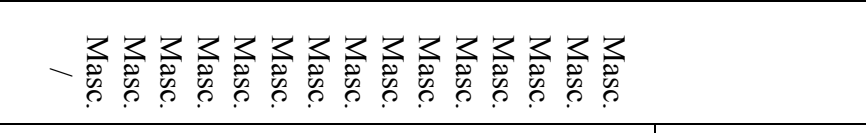 } & $\frac{z}{\frac{z}{0}}$ & & \\
\hline 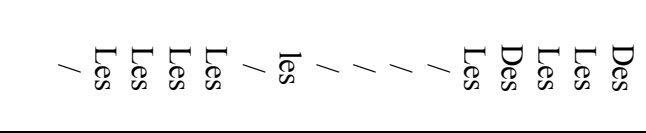 & 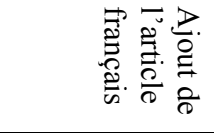 & \multirow{7}{*}{ 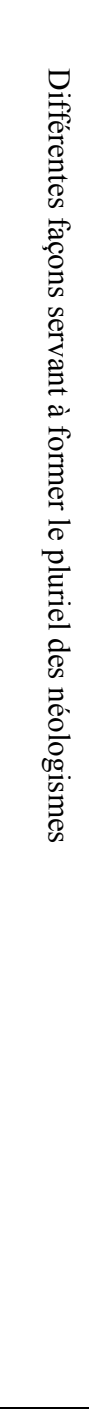 } & \multirow{7}{*}{ 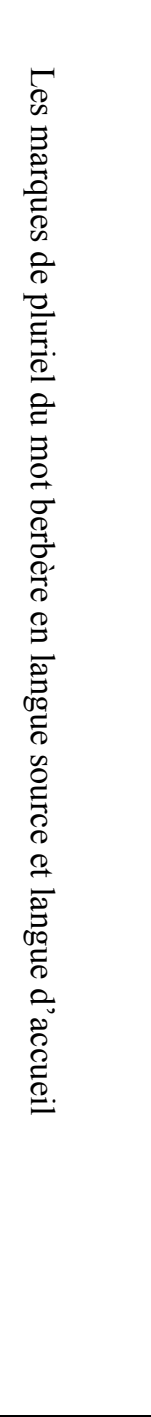 } & \\
\hline 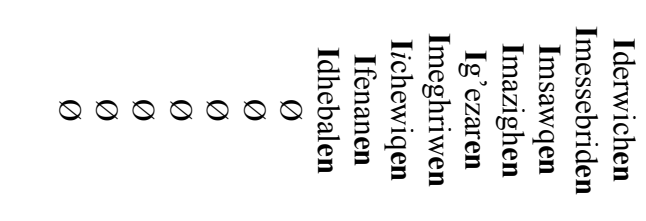 & 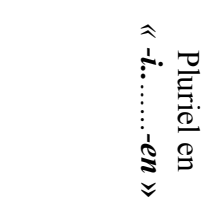 & & & \\
\hline 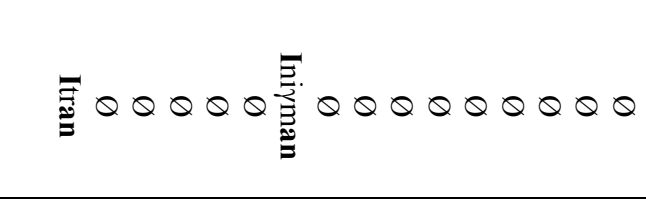 & 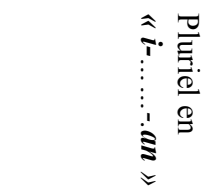 & & & \\
\hline$Q Q Q Q Q \underset{\stackrel{D}{D}}{\bar{D}} Q Q Q Q Q Q Q Q Q Q$ & 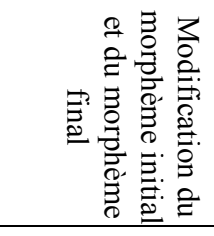 & & & \\
\hline 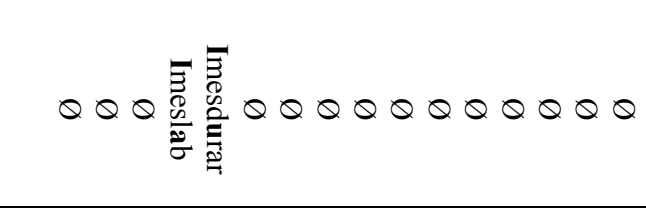 & 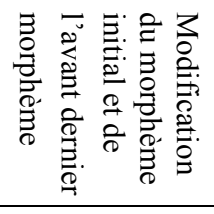 & & & \\
\hline 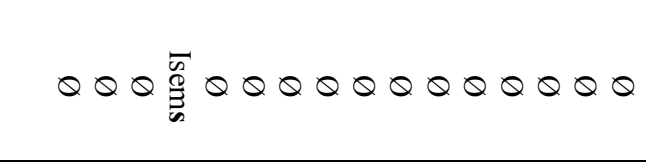 & 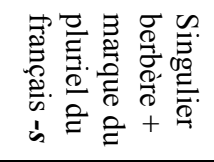 & & & \\
\hline 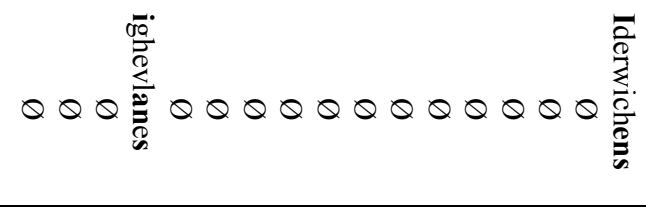 & 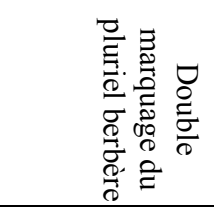 & & & \\
\hline
\end{tabular}




\section{B. Les noms féminins berbères}

Les noms féminins berbères sont peu nombreux dans notre répertoire lexical. La formation des noms de genre féminin en berbère se fait de différentes façons :

- Les noms féminins formés sur le modèle «t $t$-............t »

Par le biais de l'ajout d'un $\boldsymbol{t}$ - initial et $\boldsymbol{t} \boldsymbol{t}$ final aux mots sont formés des féminins de mots comme :

Taqcict « fille » formé sur la base du masculin aqcic « garçon »

La plus grande majorité des noms féminins singuliers contenus dans notre corpus sont formés sur ce modèle ; nous citerons comme exemples :

Tajmaât [tajemazt] qui vient de la racine berbère JM $\xi$ qui signifie «assemblée de quartier ou de village », comme l'explicite le contexte :

La tajmaât d'Aguentour destitue son «amin ». Vendredi dernier, la tajmaât du village de Tinessouine (Aguentour) s'est prononcée à la faveur de son assemblée hebdomadaire contre l'initiative des notables qu'elle impute au FFS. La tajmaât assure le nouveau chef du comité de toute sa solidarité. Cette dernière allusion indique de manière diplomatique que l'ancien «amin» du village a été destitué ». ( $L a$ Dépêche de Kabylie, 03/03/2004).

Taqvoucht [taqvuct] est aussi formé sur le même modèle. Il est le féminin de aqvouch [aqvuc], et singulier de tiqvoucin, et signifie «petite cruche d'argile cuite » dans :

Dans le coin de la Kabylie, on ne risque pas de mourir de faim tant l'hospitalité du aârch est conservée et l'Etat d'accueil réservé aux étrangers des plus chaleureux. Ici, il est devenu notoirement connu de se servir un repas très prisé, composé d'iquil (lait caillé) dans une taqevoucht (une petite cruche d'argile cuite) accompagné d'une galette et d'un tvak inighmane (figues sèches). (La Dépêche de Kabylie, 14/01/2005).

C'est de cette façon que sont formés tardast « mesure entre le pouce et l'auriculaire écartés », thassewiq $\underline{t}$ " marché organisé la veille des grandes fêtes de l'aïd », tahayek $\underline{t}$ " couverture », thakhelouit $\underline{\text { }}$ "retraite », $\underline{t a d j e b a n} \underline{t}$ « tombe ».

Il faut signaler qu'en plus du fait qu'ils sont féminins, les noms formés par addition de cette marque sont, dans certains cas, compris comme diminutif ou augmentatif dans :

Axxam « maison »----------taxxamt « petite maison ».

Ou encore comme marque véhiculant un sens péjoratif dans :

Argaz « homme »-----------targazt « homme efféminé ».

\section{- Les autres façons de former le féminin berbère}

C'est la formation d'un féminin suite à un changement radical de signifiant. Celle-ci se fait sur la base d'un signifiant autre que celui de départ. Il s'agit en effet du procédé de supplétisme, de la manière suivante : 
L'autre façon de former le féminin en berbère n'a rien à voir avec l'ajout d'une quelconque marque. Dans ce type, nous inscrivons des mots comme: ouaâda, jiff $\boldsymbol{a}$, horma, khoulou $\boldsymbol{a}$, baraka . Ces derniers représentent, à l'origine, des emprunts à l'arabe n'ayant pas subi, sur le plan formel, de changements perceptibles. Le - $\boldsymbol{a}$ final, marque du féminin arabe, est aussi marque du féminin des mots berbères comme thal $\underline{a}$ 《 source », tass $\underline{a}$ 《 foie », naâm $\underline{a}$ 《 couscous ».

Les noms berbères peuvent avoir également des terminaisons variables, chose constatée à partir des

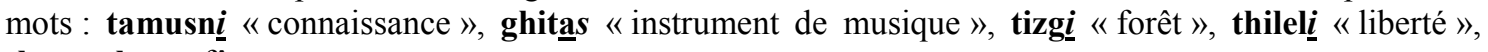
thameghra « fềte ».

\section{b. Le pluriel des noms féminins berbères}

De l'analyse des mots recueillis, se révèlent pour le pluriel des noms féminins berbères les marques suivantes:

- L'ajout de la particule -in

taqdimt $t$ « vieille chose »-------------tiqdimin.
taghrift « crêpe »---------- tighrifin.
taghourfe $t$ « chambre $»--------$ thgourfath $\underline{\underline{n}}$.

Et c'est sur le même modèle que sont formés : tikourvavine, thimeçdhathine, tikherbichine.

\section{- Formation de pluriel par supplétisme}

D'autres mots sont marqués au pluriel par un bouleversement complet du signifiant. Ce phénomène appelé supplétisme est constaté à partir de mots comme :

Tịmouchouh $\underline{a}$ [timucuha] qui signifie «conte, histoire», du singulier féminin tamacahut « conte, histoire »

Tiggur $\underline{a}$ du singulier taggurt « porte ».

\section{- Pluriel formé sur singulier + -s marque du pluriel français}

C'est par addition d'un $-\boldsymbol{s}$ (marque du pluriel français) au singulier berbère féminin que sont formés les

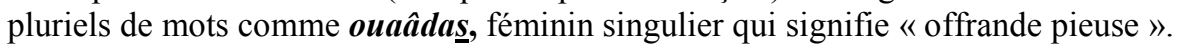

\subsubsection{La formation de dérivés}

De l'ensemble des mots recueillis, nous comptons une unité seulement ayant subi le processus de dérivation suffixale. Ainsi, le mot aârchiste est un dérivé formé à partir du mot berbère arch [1вeṛc] « tribu ». Ce nom fut utilisé lors des évènements de la Kabylie pour nommer le mouvement ayant prôné le dialogue avec l'Etat. Un aârchiste est donc « un partisan du mouvement ».

\subsection{L'adaptation phonologique}

L'adaptation phonologique se traduit par la francisation des phonèmes en leur trouvant des équivalents en langue française. Ceci provoque une variation dans la graphie des mots :

$[\varepsilon]$ est transcrit en $\hat{a}$ dans na $\underline{a} \mathbf{m a}$ [ne $\boldsymbol{\varepsilon m a}]$ qui signifie « couscous $»^{11}$

[c] est trascrit ch dans taqevoucht [taqvouct] « cruche en terre cuite»,

[y] est transcrit en $g h$ dans amghar [amyar] « vieux ».

En outre, pour le même mot nous repérons deux graphies différentes de la manière suivante :

[ḍ] $\longrightarrow \mathbf{d} / \mathbf{d h}$ dans Idhebalen/ id debalen « musiciens». 
[J] $\longrightarrow \mathbf{j} / \mathbf{d j}$ dans Tadjimaât/ tạjmaât «assemblée de village ».

$[\mathbf{t}] \longrightarrow \mathrm{t} / \mathrm{th}$ dans $\underline{T h a l a / t a l a} \ll$ source ».

\subsection{L'insertion contextuelle comme marque d'adaptation}

En plus de l'adaptation morphologique et phonologique, l'emprunt bénéficie aussi d'une insertion contextuelle qui se traduit par le marquage des rapports entre le mot et ceux qui le précèdent et le suivent dans l'énoncé. Les articles, adjectifs et formes conjuguées sont autant d'éléments qui accompagnent l'emprunt et permettent de résoudre le problème quant à la précision du genre et du nombre de ces mots.

Accord adjectif / nom :

*La traditionnelle thassewiqt.

L'accord de l'article ( $L a)$ et de l'adjectif (traditionnelle) qui précèdent le mot thassewiqt nous révèle que le mot est féminin.

*L'ancien amin du village.

L'adjectif ancien s'accorde avec le nom amin et nous renseigne sur le genre masculin de celui-ci.

Accord verbe/ nom :

*On ne croyait pas dans la puissance des taleb qui écrivaient des harouz contre tous les maux possibles.

L'accord du déterminant (des) et du verbe (écrivaient) avec le mot taleb, nous renseigne sur genre masculin de celui-ci.

Reprises anaphoriques :

*Et si pendant une certaine période idhebalen furent supplantés par d'autres groupes «plus à la mode », ils reprirent du terrain ces dernières années.

Dans cet extrait, la reprise anaphorique à partir du pronom personnel ils (marquant le pluriel masculin) nous renseigne sur le genre masculin et le nombre pluriel du mot idhebalen « troupe musicale ».

\section{Les différentes dimensions de l'emprunt}

L'emprunt du français au berbère en Algérie assure des fonctions diverses. Le français sert aux cotés du berbère dans la communication et l'intercompréhension entre les locuteurs. L'usage du berbère dans les contextes français sert à compenser un manque, donc l'absence de mots correspondant à la réalité décrite. Mais, dans le cas où le français dispose d'équivalent, le locuteur, estimant que le mot français n'est pas assez expressif par rapport à son équivalent en berbère, recourt à l'usage du mot berbère ${ }^{12}$. En effet, le recours aux emprunts berbères se fait en vue de décrire fidèlement des aspects de la vie quotidienne, parmi lesquels on trouve la volonté véhiculer l'identité berbère dans toutes ses dimensions comme: l'ethnie, la religion, les pratiques socio-culturelles.

Des emprunts compris dans notre corpus s'est dégagée une typologie ${ }^{13}$ qui repose sur la répartition des unités en différents domaines reflétant les différents aspects de la vie du locuteur berbère algérien. Elles désignent des pratiques religieuses, sociales, comportementales et gastronomiques témoignant de la valeur historique et de la richesse culturelle de ce pays. Des dimensions qui se dégagent de notre corpus, nous avons : 
- La dimension religieuse : l'aïd tamokrante, cheikh, taleb, , awal Rebbi, lemqam, axxam Rebbi...

- La dimension diatopique : tamurt, taddart, tasswiqt, thamaâmart, tadjebant, tardast, thala, tighourfathin, akham tadert...

- L'art culinaire : tihbal n'tmallalin, lesfendj, aghroum el koucha, khefaf, al asvan, aqedid, aviçar, iquil, inighman, thazart, tghrifin...

- Les métiers ou responsabilités : seggad, ag'eldoun, qadi, bokato, ig'ezaren, amghar taddart, ag'ellid, iderwichen, idhebbalen, ifenanen, imeghriwen...

- Pratiques, fêtes rituels et croyances : argaz, thakhelouit, tamhuqranit, timechret, ameddeh, timeghriwin, takoubelt, isefra, tavyitha, louziaâ, ichewiqen, ourar, asensi...

- $\quad$ Etats et sentiments : rahma, n'neya, vouvrak, horma, nif...

\section{Conclusion}

L'emprunt est un moyen de renouvellement lexical utilisé de manière abondante dans la presse écrite en Algérie. Dans cet échange ou transfert de mots, le français joue le rôle de langue d'accueil, alors que le berbère est la langue source à partir de laquelle le français se ressource en vue de s'adapter à ce milieu qui n'est pas le sien. Dans ce transfert, les mots empruntés assument tantôt les marques de genre et de nombre de la langue source et tantôt celles de la langue d'accueil. L'analyse de ces marques permet de retracer le parcours de l'emprunt et de situer son degré d'éloignement de la langue source et celui de son rapprochement de la langue d'accueil. Le français en Algérie vit et change en fonction des mutations extralinguistiques et s'adapte aux besoins multiples des locuteurs francophones.

\section{Références bibliographiques}

Chérif, S. (2004). Autour de la co-officialité de l'amazigh et de l'arabe. In A. Quefféléc / Y. Derradji / D. Morsly (éds.), Des langues et des discours en question. Les Cahiers du SLADD, SLADD Éditions, 71-85.

Cheriguen, F. (2002). Les mots des uns, les mots des autres. Le français au contact de l'arabe et du berbère. Alger : Casbah Éditions.

Dallet, J.-M. (1982). Dictionnaire Kabyle-Français. Parler des At Mangellat Algérie. Paris : SELAF.

Derradji, Y. (1995). L'emploi de la suffixation -iser, -iste, -isme, -isation dans la procédure néologique du français en Algérie. In A. Quefféléc / F. Benzakour / Y. Cherrad-Bencherfa (éds), Le français au Maghreb. Aix-enProvence : PUF, 111-119.

Derradji, Y. (2004). La langue française en Algérie : particularisme lexical ou norme endogène ? In Des langues et des discours en question. In A. Quefféléc / Y. Derradji / D. Morsly (éds.), Des langues et des discours en question. Les Cahiers du SLADD, SLADD Éditions, 14-23.

Dourari, A. (2003). Les malaises de la société algérienne. Crise de langues et crise d'identité. Alger: Casbah Éditions.

Dubois, J. et al. (1973). Dictionnaire de linguistique. Paris : Larousse.

Grandguillaume, G. (1998). Arabisation et légitimité politique en Algérie. In S. Chaker (éd.), Langue et pouvoir. De l'Afrique du Nord à l'Extrême-Orient, EDISUD, 17-23.

Laroussi, F. (éd.) (1997). Plurilinguisme et identités au Maghreb. En quels termes les dire ? Plurilinguisme et identités au Maghreb, Rouen : Publication de 1'Université de Rouen n 233, 21-31.

Louanouci, M. (1998). Les Berbères et leur langue : le cas de l'Algérie. In C. Canut (éd.), Imaginaires linguistiques en Afrique. Actes du Colloque de l'INALCO. Attitudes et représentations et imaginaire linguistique en Afrique. Quelles notions pour quelles réalités ? 9 novembre 1996. Paris : L'Harmattan, 131-146.

Maingueneau, D. (1996). Les termes clefs de l'analyse du discours. Paris : Éditions du Seuil.

Maougal, M.-L. (1996). Quel devenir pour quelle culture française en Algérie dans l'Algérie du XXI siècle ? In M. Gontard (éd.), Regard sur la francophonie, Rennes : Presses Universitaires de Rennes, 105-113.

Merzouk, S. (2012). Analyse des phénomènes néologiques : emprunt, dérivation et siglaison en situation de contact entre français, arabe et berbère dans la presse écrite en Algérie, Thèse de Doctorat, Université de Bejaia. 
Mitchell, S. (1996). Francophonie et nationalisme linguistique en Algérie à partir du processus de décolonisation. In M. Gontard (éd.), Regards sur la francophonie, Rennes : Presses Universitaires de Rennes, 99-104.

Myers-Scotton, C. (2002). Bilingual Encounters and Grammatical Outcomes. Oxford : Oxford University Press.

Poplack, S. (1988). Conséquences linguistiques du contact des langues : un modèle d'analyse variationniste. Langage et société 43 (Conférence plénière du colloque de Nice : Contacts de langues : quels modèles), 23-48.

Queffélec, A. et al. (dir.). (2002). Le français en Algérie, lexique et dynamique des langues. Paris : Duculot.

Zeghidour, S. (1994). Le rôle et le devenir du français en Algérie. In S. Abou / K. Haddad (éds.), Une francophonie différentielle, Paris : L'Harmattan, 371-377.

\section{Sitographie}

Ben Mustapha, H. (2007). Le code-switching observé chez les diplômés de l'université en Tunisie : étude linguistique et sociolinguistique, Université de Manouba, Tunisie.

$<$ http://www.academia.edu/4800735/Le codeswitching observe chez les diplomes de luniversite en Tunisie $>$ (consulté le 07 mars 2014)

Bourhis, R. Y. / Lepicq, D. / Sachdev, I. (2000). La psychologie sociale de la communication multilingue. Divers Cité Langues. En ligne. Vol. V. <http://www.teluq.uquebec.ca/diverscite> (consulté le 07 mars 2014)

Derradji, Y. Le français en Algérie, langue emprunteuse et empruntée. $<$ www.unice.fr/ILF-CNRS/ofcaf/13/derradji.html> (consulté le 25 octobre 2013).

Constitution de 1963 : <http://www.conseil-constitutionnel.dz/Constituion1963_2.htm> (consulté le 11 novembre 2013).

Grandguillaume, G. (2004). L'arabisation au Maghreb. Revue d'Aménagement linguistique, Office Québécois de la langue française, $\mathrm{n}^{\circ} 107,15-40$.

$<$ http://www.ggrandguillaume.fr/titre.php?recordID=51> (consulté le 26 février 2014).

Grandguillaume, G. (2010). L’Algérie pays francophone ? $<$ http://www.ggrandguillaume.fr/titre.php?recordID $=45>$ (consulté le 23 février 2014).

Gumperz, J. (1982). Discourse strategies. Cambridge : Cambridge University Press. $<\mathrm{http}: / /$ creoles.free.fr/Cours/alternance.htm $>$ (consulté le 03 mars 2014).

Mabrour, A. L'alternance codique arabe/français : emplois et fonctions, Constellations francophones, Publifarum, $\mathrm{n}^{\circ}$ $7,20 / 12 / 2007$

$<$ http://publifarum.farum.it/ezine_articles.php?id=67> (consulté le 03/03/2014).

Taleb Ibrahimi, K. L'Algérie : coexistence et concurrence des langues. L'Année du Maghreb [en ligne], I| 2004, mis en ligne le 08 juillet 2010.

[URL : http://anneemaghreb.revues.org/305; DOI : 10.4000/anneemaghreb.305] (consulté le 21 février 2014).

\footnotetext{
${ }^{1}$ L'arabe est réparti, selon Taleb Ibrahimi, en différentes variétés. D'abord, l'arabe classique qui correspond au registre le plus normé de la langue arabe, se rapprochant du modèle idéal que représente le texte coranique. «La langue arabe classique entretient donc un lien privilégié avec l'islam : emblème de l'identité musulmane, elle est ressentie comme telle par les musulmans depuis des siècles. Son lien avec l'islam la fait participer de sa légitimité religieuse et par dérivation politique, puisque l'islam est la Loi d'Allah à laquelle aucune autre ne peut être opposée pour les croyants » (Grandguillaume, 2010). Ensuite, l'arabe standard ou moderne assurant l'intercommunication entre les différents pays arabophones. L'arabe, langue de scolarisation utilisée également dans les productions intellectuelles, et est aussi la langue des médias. Enfin, l'arabe spontané, à usage exclusivement oral, est essentiellement utilisé en milieu familial, à la télévision et à la radio.

${ }^{2}$ «Le français colonial en Algérie est une réalité linguistique mais aussi culturelle, qui tire son originalité de l'interpénétration socioculturelle et de la situation de contact des langues de groupes qui prévalaient après la conquête militaire » (Queffélec, $2002: 28$ ).

${ }^{3}$ Cité par Maïna F. La langue algérienne existe-t-elle vraiment? Quelques articles sur l'enseignement et les langues en Algérie, publié le 6 Juin 2013 par Pour une Algérie démocratique et sociale, [URL : http://mohbelkacimi.overblog.fr/article-quelques-articles-sur-l-enseignement-et-les-langues-en-algerie-118324490.html]

${ }^{4}$ Kamel Daoud, écrivain, chroniqueur au Quotidien d'Oran, et auteur de plusieurs ouvrages dont : Raïna raïkoum, Dar El Gharb, Oran, (2002), Ô Pharaon, Dar El Gharb, Oran, (2005), Minotaure 504 (nouvelles), Sabine Wespieser, Paris (2011).
} 
${ }^{5}$ Cité par Maïna F. La langue algérienne existe-t-elle vraiment ? Quelques articles sur l'enseignement et les langues en Algérie, publié le 6 Juin 2013 par Pour une Algérie démocratique et sociale, [URL : http://mohbelkacimi.overblog.fr/article-quelques-articles-sur-l-enseignement-et-les-langues-en-algerie-118324490.html].

${ }^{6}$ Militant pour la cause berbère, auteur de nombreux romans tels que : La colline oubliée (1952), Le sommeil du juste (1955), L'Opium et le Bâton (1965) et des ouvrages de grammaire berbère : Précis de grammaire berbère (1987), Tajjerumt n'tamazight (1976).

${ }^{7}$ Suite à un vote en avril 2002, les membres du Parlement algérien votent à l'unanimité pour une modification de la Constitution, en conférant au berbère le statut de langue nationale. L'article 3 bis est additionné à l'article 3 de la Constitution décrétant que «le tamazight est également langue nationale. L'Etat œuvre à sa promotion et à son développement dans toutes ses variétés linguistiques en usage sur le territoire national ».

${ }^{8}$ Citée par Ben Mustapha, H. (2007). Le codes-switching observé chez les diplômés de l'université en Tunisie : Etude linguistique et sociolinguistique, Université de Manouba, Tunisie, p. 40 [http://www.academia.edu/4800735/Le_codeswitching_observe_chez_les_diplomes_de_luniversite_en_Tunisie], (consulté le 07 mars 2014).

${ }^{9}$ Cité par Asselah-Rahal, S. (1993). Pratiques linguistiques trilingues (arabe, kabyle, français) chez les locuteurs algériens, Thèse de Magister, Université d'Alger, p. 89.

${ }^{10}$ Les mots qui figurent entre crochets représentent la transcription en berbère des mots contenus dans notre corpus.

${ }^{11}$ Le sens attribué aux mots est celui qui ressort de leur contexte d'utilisation et qui n'est pas forcément celui qu'ils recouvrent dans le dictionnaire.

${ }^{12}$ Situation constatée dans le cadre du bilinguisme de compétence, quand le sujet bilingue, ayant la maîtrise des deux systèmes linguistiques, procède au choix d'un mot et non d'un autre, choix reposant sur la force expressive ou pas de l'unité lexicale.

${ }^{13}$ Inspirée de l'analyse de D. Morsly (1995) «El Watan, El Moujahid, Algérie-Actualités, El-Djeich, Liberté, Le Matin... La presse algérienne de langue française et l'emprunt à l'arabe », Plurilinguismes, 9-10, 35-53. 